Artículo

\title{
Comportamiento genético para componentes de rendimiento en una población de maíz enano
}

\author{
Eduardo Hernández Alonso ${ }^{\S}$ \\ Humberto De León Castillo \\ Instituto Mexicano del Maíz Dr. Mario E. Castro Gil-Universidad Autónoma Agraria Antonio Narro. \\ Calzada Antonio Narro núm. 1923, Buenavista, Saltillo, Coahuila, México. (hleonc62@hotmail.com). \\ ${ }^{\S}$ Autor para correspondencia: eduardoalonso0821@gmail.com.
}

\section{Resumen}

Los componentes de rendimiento son variables importantes para realizar selección indirecta hacia la productividad. En maíz, el rendimiento está correlacionado con la longitud de la mazorca, el peso hectolítrico y el tamaño de grano. En esta investigación se analizaron los resultados de un dialélico que fue sometido a ensayos de rendimiento en dos localidades durante 2016, formado con cinco líneas representativas de una población de maíz de porte enano. Lo anterior, para estimar los efectos genéticos y la heredabilidad del rendimiento mediante tres componentes del rendimiento, con la finalidad de definir la estrategia de mejoramiento a utilizar en cada componente. El análisis genético se realizó mediante el modelo II, método III de Griffing. Los resultados mostraron, que la varianza del tipo aditivo gobierna el comportamiento de las variables de longitud de mazorca y rendimiento. El tipo de acción génica no aditiva es responsable del comportamiento fenotípico del peso hectolítrico, mientras que los efectos recíprocos (materno y no maternos) son los que más influyen para la expresión fenotípica de profundidad de grano. Además, se encontraron valores altos de heredabilidad con un estimado de 0.56 para longitud de mazorca y 0.58 para rendimiento, mientras que para peso hectolítrico y profundidad de grano la heredabilidad encontrada es baja 0 y 0.07, respectivamente. Se sugiere la utilización del esquema de mejoramiento de hermanos completos para longitud de mazorca y rendimiento, para peso hectolítrico y profundidad de grano es preferible utilizar progenies endogámicas.

Palabras clave: análisis dialélico, efectos genéticos, heredabilidad.

Recibido: mayo de 2021

Aceptado: julio de 2021 


\section{Introducción}

México es partícipe importante en la producción mundial de maíz ocupando el $8^{\circ}$ lugar con una producción de 27228242 t en una superficie de 7157586 ha. Aun así, es deficitario en la producción de maíz, lo que impone la necesidad de adquirir significativas cantidades en el mercado externo, anualmente importa 17 millones de toneladas de maíz debido al bajo rendimiento de este cereal a nivel nacional que es de $4.07 \mathrm{t} \mathrm{ha}^{-1}$ (SIAP, 2020).

El aumento de la producción agrícola orienta a la investigación a alcanzar la producción en mayor cantidad por unidad de superficie. Debido a esto, el uso de maíces enanos surge como una alternativa que confiere las ventajas de: acomodar más plantas por hectáreas sin reducir el tamaño de la mazorca lo que le permitirá al agricultor levantar mayor rendimiento por unidad de superficie, mejor aprovechamiento de la energía solar al tener la disposición vertical de sus hojas arriba de la mazorca, una gran adaptación a la mecanización del cultivo ya que su porte bajo mejora la facilidad de acceso de maquinaria para la aplicación de agroquímicos como son los nutrientes foliares, herbicidas, control químico de plagas y enfermedades, etc., mayor tolerancia a los acames por su reducida altura y buen sistema radicular; respuesta en la siembra de híper-densidades, además se adapta con facilidad a las diferentes localidades de la regiones subtropicales tolerando las limitaciones ocasionales de no disponibilidad de agua.

En el cultivo de maíz, es común que al realizar prácticas de mejoramiento directamente para rendimiento también se mejoren otras variables que están correlacionadas positivamente con esta variable, como son: la longitud de mazorca, el peso hectolítrico o volumétrico y la profundidad del grano, dichas variables son conocidas como componentes de rendimiento. Estas características son importantes, porque al mejorarlas de manera directa o indirecta aumentan la productividad por unidad de superficie.

El rendimiento de mazorca y de grano correlacionan positivamente con las variables que comprenden los componentes de rendimiento; particularmente la mejor expresión de rendimiento se da por tener una mayor longitud de mazorca (Wong et al., 2007; Díaz et al., 2009; Pecina et al., 2011). Por otro lado, Arellano et al. (2010), reportan altos valores de correlación con peso hectolítrico, mientras que Borrás y Otegui (2001), mencionan que el tamaño de grano, entre más grande, permite una mayor expresión en el rendimiento.

Actualmente se requiere que el maíz mantenga al máximo su capacidad productiva, para esto es deseable conocer el tipo de acción génica que gobierna cada carácter y así, incorporar con mayor facilidad características agronómicas que ofrezcan una ventaja competitiva. En este sentido, es necesario la caracterización de los fenotipos a través de técnicas que permitan determinar los efectos genéticos involucrados en los materiales de estudio (Wong et al., 2007).

El análisis dialélico es una herramienta que permite estimar la cuantía de la varianza aditiva y la de dominancia en el comportamiento de las variables; asimismo, estiman los efectos de aptitud combinatoria, efectos recíprocos y maternos de los progenitores, también permite conocer la heterosis de los cruzamientos y la heredabilidad del caracter (De León et al., 2005; Coutiño et al., 2010). 
La heredabilidad $\left(\mathrm{h}^{2}\right)$, es considerada como el parámetro fundamental para la selección, puesto que determina la cantidad de variación total encontrada en una característica que es atribuida al efecto directo de los genes (varianza aditiva); es decir, a la herencia (Ruales et al., 2007).

En este trabajo de investigación, a partir de un análisis dialélico entre cinco líneas representativas de una población de maíz de porte enano, se estimó la aptitud combinatoria general, aptitud combinatoria específica, efectos recíprocos, efectos maternos y efectos no maternos, para generar conocimiento de que tipo de acción génica es la que controla cada componente del rendimiento y la heredabilidad, para definir qué estrategia de mejoramiento utilizar para la siguiente generación de mejoramiento.

\section{Materiales y métodos}

\section{Material genético}

En la presente investigación se utilizaron las cruzas directas y recíprocas entre cinco líneas de maíz de porte enano con avanzado grado de endogamia, representativas de la población de maíz enano y pertenecientes al programa de mejoramiento del Instituto Mexicano del Maíz (IMM) Dr. Mario E. Castro Gil de la Universidad Autónoma Agraria Antonio Narro (UAAAN). Las líneas evaluadas fueron: LE1= línea enana 1; LE2= línea enana 2; LE3= línea enana 3; LE4= línea enana 4 y LE5= línea enana 5.

\section{Diseño de apareamiento}

El sistema de apareamiento empleado fue el método III de Griffing (1956), el cual permitió generar cruzas directas y recíprocas a partir de $\mathrm{p}$ líneas progenitores, originando $\mathrm{p}$ ( $\mathrm{p}-1$ ) genotipos diferentes. Los cruzamientos del dialélico entre las líneas enanas se realizaron en el campo directo del Instituto Mexicano del Maíz, obteniendo las cruzas requeridas.

\section{Condiciones de desarrollo}

El ensayo de rendimiento se realizó bajo un diseño de bloques al azar con dos repeticiones por ambiente, en el primer ambiente se sembró el 03 de marzo y en el segundo ambiente se sembró el 16 de abril, ambos ubicados en la localidad de Buenavista, Saltillo, Coahuila, a una latitud norte de $25^{\circ} 20^{\prime}$, longitud oeste $101^{\circ} 01^{\prime}$ y una altitud de $1750 \mathrm{~m}$, durante el año 2016. La unidad experimental consistió en un surco de $3.84 \mathrm{~m}$ de largo, donde se sembraron 33 semillas espaciadas a $11.6 \mathrm{~cm}$, el espaciamiento entre surcos fue de $0.8 \mathrm{~m}$.

Durante el manejo agronómico se realizó una fertilización con una dosis de 240-100-100 que corresponden a las unidades de nitrógeno $(\mathrm{N})$, fósforo $(\mathrm{P})$ y potasio $(\mathrm{K})$ respectivamente, aplicando la mitad del nitrógeno y todo el fosforo y potasio al momento de la siembra, el resto de la fertilización se aplicó en el primer cultivo. El control de malezas se realizó de forma química aplicando el ingrediente activo (atrazina) con el nombre comercial Gesaprim Calibre 90 de la empresa Syngenta. Para el control de plagas se aplicó el producto Proclaim 5 GS, con ingrediente activo (benzoato de emamectina) de la empresa Syngenta, además se aplicó el producto Topgard 75 WP con ingrediente activo (cyromazina 75\%) de la empresa Koor Intercomercial, SA. 


\section{Componentes de rendimiento}

Los componentes del rendimiento se determinaron con el valor promedio de 10 mazorcas representativas, obtenidas de cada unidad experimental, las variables evaluadas fueron: la longitud de mazorca se obtuvo de la distancia comprendida desde la base hasta el ápice de la mazorca superior $(\mathrm{cm})$. Peso hectolítrico el cual se obtuvo de una muestra de $250 \mathrm{~g}$ por parcela, se estimó con el aparato mini GAC plus de la empresa Dickey-John sinc, 1966 la información se reportó en volumen. Profundidad de grano se obtuvo con un Vernier de precisión (Steren, HER-411), este dato se generó del diámetro central de la mazorca menos el diámetro del olote dividido entre dos (cm). El rendimiento se reportó en $\mathrm{t} \mathrm{ha}^{-1}$ de mazorca y se ajustó al $15.5 \%$ de humedad.

\section{Análisis estadístico y genético}

El análisis de componentes genéticos y la estimación de heredabilidad se realizó en dos ambientes mediante modelo II el método III de Griffing (1956), con la rutina Diallel-SAS05 propuesto por Zhang et al. (2005). Cuyo modelo genético se presenta a continuación: $Y_{i j k l m}=\mu+A_{i}+R_{j(i)}+g_{k}+$ $g_{l}+s_{k l}+m_{m}+A g_{i k}+A g_{i l}+A s_{i k l}+A m_{i m}+\mathscr{E}_{i j k l m}$. Donde: $Y_{i j k l m}=$ variable de respuesta; $\mu=$ efecto de la media general; $A_{i}=$ efecto del $i$-ésimo ambiente; $R_{j(i)}=$ efecto de la $j$-ésima repetición dentro del $i$-ésimo ambiente; $g_{k}=$ efecto de la aptitud combinatoria general del padre $k ; g_{l}=$ efecto de la aptitud combinatoria general del padre $l ; s_{k l}=$ efecto de la aptitud combinatoria específica de los padres $k$ y $l ; m_{m}=$ efecto recíproco; $A g_{i k}=$ efecto de la interacción entre el $i$-ésimo ambiente y la aptitud combinatoria general del padre $k ; A g_{i l}=$ efecto de la interacción entre el $i$-ésimo ambiente y la aptitud combinatoria general del padre $l ; A s_{i k l}=$ efecto de la interacción entre el $i$-ésimo ambiente y la aptitud combinatoria específica de los padres $k$ y $l ; A m_{i m}=$ efecto de la interacción entre el $i$ ésimo ambiente y el efecto recíproco y; $\mathscr{E}_{i j k l m}=$ error experimental.

El porcentaje de aportación de los efectos de aptitud combinatoria general (ACG), aptitud combinatoria específica (ACE), efectos recíprocos (EREC), efectos maternos (EMAT) y efectos no maternos (ENMAT) se calculó con base a la proporción de la suma de cuadrados de la fuente, con respecto a la suma de cuadrados total.

A partir de la esperanza de cuadrados medios (Cuadro 1), se calculó $\sigma_{A}^{2}, \sigma_{D}^{2}, \sigma_{F}^{2}$ y la heredabilidad en sentido estrecho. Donde: $\sigma_{A}^{2}=2 \sigma_{a c g}^{2} ; \sigma_{D}^{2}=\sigma_{a c e}^{2} ; \sigma_{F}^{2}=\left(2 \sigma_{a c g}^{2}\right)+\left(\sigma_{a c e}^{2}\right)+\left(2 \sigma_{\text {erec }}^{2}\right)+$ $\left(2 \sigma_{\text {acg } x a m b}^{2} /_{a m b}\right)+\left(\sigma_{\text {ace } x a m b / a m b}^{2}\right)+\left(2 \sigma_{\text {erec } x a m b / a m b}^{2}\right)+($ error $/$ rep $* a m b) \cdot \mathrm{h}^{2}=\frac{\sigma_{A}^{2}}{\sigma_{F}^{2}}$

Cuadro 1. Descripción del análisis genético combinado a través de ambientes del modelo II, método III de Griffing.

\begin{tabular}{cccc}
\hline Fuentes de variación & $\mathrm{gl}$ & $\mathrm{CM}$ & ECM \\
\hline Ambientes (Amb) & $\mathrm{a}-1$ & & \\
Repeticiones (Rep)/Amb & $\mathrm{a}(\mathrm{r}-1)$ & & \\
Híbrido (Hib) & $\mathrm{p}(\mathrm{p}-1)-1$ & $\mathrm{M}_{h}$ & $\sigma_{e}^{2}+\mathrm{r} \sigma_{h y}^{2}+\mathrm{ra} \sigma_{h}^{2}$ \\
$\mathrm{ACG}$ & $\mathrm{p}-1$ & $\mathrm{M}_{g}$ & $\sigma_{e}^{2}+2 \mathrm{r} \sigma_{s y}^{2}+2 \mathrm{ra} \sigma_{s}^{2}+2 \mathrm{r}(\mathrm{p}-2) \sigma_{g y}^{2}+2 \mathrm{ra}(\mathrm{p}-2) \sigma_{g}^{2}$ \\
$\mathrm{ACE}$ & $\mathrm{p}(\mathrm{p}-3) / 2$ & $\mathrm{M}_{s}$ & $\sigma_{e}^{2}+2 \mathrm{r} \sigma_{s y}^{2}+2 \mathrm{ra} \sigma_{s}^{2}$ \\
EREC & $\mathrm{p}(\mathrm{p}-1) / 2$ & $\mathrm{M}_{r}$ & $\sigma_{e}^{2}+2 \mathrm{r} \sigma_{r y}^{2}+2 \mathrm{ra} \sigma_{r}^{2}$ \\
\hline
\end{tabular}




\begin{tabular}{cclc}
\hline Fuentes de variación & $\mathrm{gl}$ & $\mathrm{CM}$ & $\mathrm{ECM}$ \\
\hline EMAT & $\mathrm{p}-1$ & $\mathrm{M}_{m}$ & $\sigma_{e}^{2}+2 \mathrm{r} \sigma_{n y}^{2}+2 \mathrm{ra} \sigma_{n}^{2}+2 \mathrm{rp} \sigma_{m y}^{2}+2 \mathrm{rap} \sigma_{m}^{2}$ \\
ENMAT & $(\mathrm{p}-1)(\mathrm{p}-2) / 2$ & $\mathrm{M}_{n}$ & $\sigma_{e}^{2}+2 \mathrm{r} \sigma_{n y}^{2}+2 \mathrm{ra} \sigma_{n}^{2}$ \\
Hib x Amb & $\mathrm{p}(\mathrm{p}-1)(\mathrm{a}-1)$ & $\mathrm{M}_{h y}$ & $\sigma_{e}^{2}+\mathrm{r} \sigma_{h y}^{2}$ \\
ACG x Amb & $(\mathrm{p}-1)(\mathrm{a}-1)$ & $\mathrm{M}_{g y}$ & $\sigma_{e}^{2}+2 \mathrm{r} \sigma_{s y}^{2}+2 \mathrm{r}(\mathrm{p}-2) \sigma_{g y}^{2}$ \\
ACE x Amb & $\mathrm{p}(\mathrm{p}-3)(\mathrm{a}-1) / 2$ & $\mathrm{M}_{s y}$ & $\sigma_{e}^{2}+2 \mathrm{r} \sigma_{s y}^{2}$ \\
EREC x Amb & $\mathrm{p}(\mathrm{p}-1)(\mathrm{a}-1) / 2$ & $\mathrm{M}_{r y}$ & $\sigma_{e}^{2}+2 \mathrm{r} \sigma_{r y}^{2}$ \\
EMAT x Amb & $(\mathrm{p}-1)(\mathrm{a}-1)$ & $\mathrm{M}_{m y}$ & $\sigma_{e}^{2}+2 \mathrm{r} \sigma_{n y}^{2}+2 \mathrm{rp} \sigma_{m y}^{2}$ \\
ENMAT x Amb & $(\mathrm{p}-1)(\mathrm{p}-2)(\mathrm{a}-1) / 2$ & $\mathrm{M}_{n y}$ & $\sigma_{e}^{2}+2 \mathrm{r} \sigma_{n y}^{2}$ \\
Error & {$[\mathrm{p}(\mathrm{p}-1)][\mathrm{a}(\mathrm{r}-1)]$} & $\mathrm{M}_{e}$ & $\sigma_{e}^{2}$ \\
\hline
\end{tabular}

$\mathrm{gl}=$ grados de libertad; $\mathrm{ACG}=$ aptitud combinatoria general; $\mathrm{ACE}=$ aptitud combinatoria específica; $\mathrm{EREC}=$ efectos recíprocos; EMAT = efectos maternos; ENMAT = efectos no maternos; $\mathrm{CM}=$ cuadrados medios; $\mathrm{ECM}=$ esperanza de cuadrados medios.

\section{Resultados y discusión}

Los resultados para la fuente de variación de ambientes (Cuadro 2) se detectó significancia ( $p \leq$ $0.05)$ en la variable de longitud de mazorca y en el peso hectolítrico $(p \leq 0.05)$. Lo que sugiere que el comportamiento promedio de los ambientes no fue estadísticamente igual, esto es atribuido a que las condiciones climáticas, edáficas y que el manejo agronómico fue diferente. Hermes et al. (2017), señala que los componentes principales de la variabilidad cuando se realizan ensayos a través de ambientes en el mismo año son: el clima, la precipitación pluvial, la altitud, ubicación geográfica y el tipo de suelo.

Cuadro 2. Cuadrados medios del análisis genético de 20 híbridos $F_{1}$ formados a partir de 5 líneas, considerando cruzas directas y recíprocas evaluadas en dos ambientes durante el 2016.

\begin{tabular}{cccccc}
\hline Fuentes de variación & gl & $\begin{array}{c}\text { Longitud de mazorca } \\
(\mathrm{cm})\end{array}$ & $\begin{array}{c}\text { Peso } \\
\text { hectolítrico } \\
(\mathrm{vol})\end{array}$ & $\begin{array}{c}\text { Profundidad de } \\
\text { grano }(\mathrm{cm})\end{array}$ & $\begin{array}{c}\text { Rendimiento } \\
\left(\mathrm{t} \mathrm{ha}^{-1}\right)\end{array}$ \\
\hline $\begin{array}{c}\text { Ambientes (Amb) } \\
\text { Repeticiones }\end{array}$ & 1 & $11.777^{*}$ & $149.331^{* *}$ & 0.499 & 1.2 \\
(Rep)/Amb & 2 & $18.855^{* *}$ & $53.839^{* *}$ & 0.983 & $37.787^{*}$ \\
Híbridos (Hib) & 19 & $6.157^{*}$ & & & \\
ACG & 4 & $19.543^{* *}$ & $8.374^{*}$ & 0.498 & $35.296^{* *}$ \\
ACE & 5 & 1.63 & $15.229^{* *}$ & 0.549 & $110.51^{* *}$ \\
EREC & 10 & 3.066 & 4.944 & 0.484 & 8.882 \\
EMAT & 4 & 2.961 & 4.644 & 0.561 & 18.417 \\
ENMAT & 6 & 3.137 & 5.145 & 0.433 & $12.731^{*}$ \\
Hib x Amb & 19 & 2.573 & $6.835^{*}$ & 0.56 & 12.688 \\
ACG x Amb & 4 & 6.416 & 8.798 & 0.65 & 15.667 \\
ACE x Amb & 5 & 1.444 & 5.203 & 0.602 & 10.141 \\
REC x Amb & 10 & 1.6 & 6.866 & 0.504 & 12.772 \\
\hline
\end{tabular}




\begin{tabular}{cccccc}
\hline Fuentes de variación & gl & $\begin{array}{c}\text { Longitud de mazorca } \\
(\mathrm{cm})\end{array}$ & $\begin{array}{c}\text { Peso } \\
\text { hectolítrico } \\
(\mathrm{vol})\end{array}$ & $\begin{array}{c}\text { Profundidad de } \\
\text { grano }(\mathrm{cm})\end{array}$ & $\begin{array}{c}\text { Rendimiento } \\
\left(\mathrm{t} \mathrm{ha}^{-1}\right)\end{array}$ \\
\hline EMAT x Amb & 6 & 3.09 & 10.748 & 0.561 & 14.073 \\
ENMAT x Amb & 6 & 0.607 & 4.278 & 0.519 & 11.905 \\
Error & 38 & 2.473 & 3.593 & 0.519 & 9.161 \\
$\begin{array}{c}\text { Total } \\
\text { Coeficiente de } \\
\text { variación } \\
\text { Media }\end{array}$ & 79 & 10.442 & 2.678 & 58.543 & 16.956 \\
\hline
\end{tabular}

$*^{* *}=$ significativo a $(p \leq 0.05)$ y $(p \leq 0.05)$ respectivamente; $\mathrm{gl}=$ grados de libertad; $\mathrm{ACG}=$ aptitud combinatoria general; $\mathrm{ACE}=$ aptitud combinatoria específica; $\mathrm{EREC}=$ efecto recíproco; $\mathrm{EMAT}=$ efecto materno; ENMAT $=$ efecto no materno.

Para la fuente de variación de híbridos la significancia se detectó en las variables de longitud de mazorca, peso hectolítrico $(p \leq 0.05)$ y rendimiento $(p \leq 0.05)$. Lo anterior, atribuible a que los híbridos exhibieron variabilidad en estos caracteres, lo que indica que al menos uno es diferente al resto, para profundidad de grano no se encontró significancia, esto se atribuye a la poca intensidad de selección que se le ha puesto a esta característica. Abbott y Pistorale (2011), mencionan también que el amplio rango de ambientes climáticos y edáficos puede provocar respuestas diferenciales del comportamiento de los genotipos.

El desglose de los cuadrados medios de los híbridos en efectos de ACG y ACE, muestra que los efectos en ACG no son estadísticamente iguales a cero, con una significancia de $(p \leq 0.05)$ para las variables de longitud de mazorca y rendimiento. Estas diferencias encontradas en ACG, se pueden atribuir a la base genética de donde se derivaron los progenitores, los cuales ha pasado por varios ciclos de selección recurrente.

Al no detectar significancia en el peso hectolítrico y profundidad de grano se sugiere que es necesario propiciar la variabilidad para estos caracteres y así, lograr incrementar la varianza. Luna et al. (2013); Haochuan et al. (2014), mencionan que tener conocimiento amplio de la base genética del germoplasma y del tipo de acción génica de los caracteres de mayor importancia económica, como el rendimiento, esto permite maximizar la varianza genética y de esta forma incrementar y fijar la frecuencia de alelos favorables en la población.

En cuanto ACE, se detectó significancia para peso hectolítrico $(p \leq 0.05)$, lo que indica que existe efecto de dominancia; es decir, la variabilidad genética no aditiva para este carácter fue importante. Kamara et al. (2020), indican que los efectos significativos de ACG y ACE implica que tanto los efectos aditivos como los no aditivos están involucrados en el gobierno de los caracteres.

El aspecto práctico del mejoramiento genético del maíz por hibridación está basado en el desarrollo de líneas endogámicas y la evaluación de su ACG y ACE para la obtención de híbridos comerciales de alto de rendimiento. Al respecto Bernardo (2001), menciona que el comportamiento de las líneas per se no proveen una buena medida de su valor en combinaciones híbridas. Por su parte, Lobato et al. (2010), indican que el desarrollo de metodologías simples, rápidas y adecuadas para evaluar nuevas líneas ha sido un problema en la generación de híbridos con alto potencial productivo. 


\section{ACG y ACE}

En el Cuadro 3 se presentan a detalle, por progenitor, los efectos de ACG para los caracteres evaluados, en los que se puede discutir lo siguiente, para realizar cruzas complementarias dentro del mismo grupo heterótico: la línea LE01 pueden ser buena donadora en longitud de mazorca y rendimiento, ya que se detectó significancia $(p \leq 0.05)$ en ambas variables. La línea LE02 muestra valores favorables $(p \leq 0.05)$ para rendimiento.

La línea LE03 tiene efectos positivos para peso hectolítrico y profundidad de grano, aunque no son significativos. La LE05 no tuvo significancia, aun así, se observan valores positivos para longitud de mazorca y rendimiento.

En los efectos de ACE (Cuadro 3), sólo 10\% presenta valores significativos. Los bajos efectos detectados de ACE se atribuye a que las líneas pertenecen al mismo grupo heterótico, esto es de suma importancia para el programa ya que la heterosis se puede explotar según De León et al. (2005) al cruzarlos con el grupo heterótico complementario de altura normal con fondo tropical.

Cuadro 3. Estimación de efectos de ACG y ACE, obtenidos a través del diseño dialélico de Griffing modelo II, método III en cuatro variables agronómicas de 10 cruzas en $F_{1}$.

\begin{tabular}{ccccc}
\hline Variables & $\begin{array}{c}\text { Longitud de mazorca } \\
(\mathrm{cm})\end{array}$ & $\begin{array}{c}\text { Peso } \\
\text { hectolítrico } \\
(\mathrm{vol})\end{array}$ & $\begin{array}{c}\text { Profundidad } \\
\text { de grano } \\
(\mathrm{cm})\end{array}$ & $\begin{array}{c}\text { Rendimiento } \\
\left(\mathrm{t} \mathrm{ha}^{-1}\right)\end{array}$ \\
\hline ACG & $1.299^{* *}$ & -0.018 & 0.186 & $2.005^{* *}$ \\
LE01 & $-0.182^{* *}$ & -0.082 & $1.3488^{* *}$ \\
LE02 & $-0.977^{* *}$ & -0.788 & 0.129 & $-2.4074^{* *}$ \\
LE03 & -0.605 & 0.166 & -0.062 & $-1.6149^{* *}$ \\
LE04 & 0.466 & -0.201 & -0.172 & $0.6684^{*}$ \\
LE05 & & & & \\
ACE & 0.643 & 1.177 & -0.106 & $-0.3804^{* *}$ \\
LE01xLE02 & -0.222 & -0.027 & 0.388 & $1.662^{* *}$ \\
LE01xLE03 & -0.241 & -0.102 & -0.123 & -0.4429 \\
LE01xLE04 & -0.179 & -1.048 & -0.159 & -0.8387 \\
LE01xLE05 & -0.046 & 0.506 & -0.141 & -0.7979 \\
LE02xLE03 & -0.448 & -1.831 & 0.104 & 0.652 \\
LE02xLE04 & -0.149 & 0.148 & 0.143 & $0.5262^{*}$ \\
LE02xLE05 & 0.314 & 0.277 & -0.122 & -0.6684 \\
LE03xLE04 & -0.046 & -0.756 & -0.125 & $-0.1712^{*}$ \\
LE03xLE05 & 0.374 & $1.656 *$ & 0.141 & 0.4837 \\
LE04xLE05 & & & & \\
\hline
\end{tabular}

$*^{* * *}=$ significativo a $(p \leq 0.05)$ y $(p \leq 0.05)$ respectivamente; LE01= línea enana 01; LE02= línea enana 02; LE03= línea enana 03; LE04= línea enana 04; LE05= línea enana 05; ACG= aptitud combinatoria general; ACE= aptitud combinatoria específica. 
Investigaciones realizadas por Reyes et al. (2004), mencionó que se espera que los cruzamientos con mayor ACE resulten al cruzar al menos dos líneas diferentes, sin importar sus efectos de ACG. En el presente estudio se encontró que progenitores con efectos bajos o negativos de ACG produjeron buenos cruzamientos. Resultados similares fueron obtenidos por Guillen et al. (2009) al cruzar progenitores con ACG negativa y obtener cruzas con valores positivos de ACE.

Al observar el comportamiento de los cruzamientos se muestra significancia en ACE para la cruza LE01x LE03 en profundidad de grano a $(p \leq 0.05)$ y al $(p \leq 0.05)$ para rendimiento (Cuadro 3$)$, siendo esta progenie de las líneas que presentaron significancia para ACG tanto positiva como negativa; dicho resultado produjo la cruza más sobresaliente para estos componentes de rendimiento.

La cruza que presentó un valor positivo con una significancia de $(p \leq 0.05)$ es LE04xLE05 (Cuadro 3 ). Esto es de suma importancia ya que el peso hectolítrico de los granos es uno de los parámetros que determinan el rendimiento; además de contribuir directamente a la sanidad de grano, rapidez de secado y resistencia ante el ataque de hongos, esta última característica es de importancia ante la problemática de sanidad de este cereal, ya que afecta directamente al rendimiento. Olán et al. (2012), mencionan que el peso hectolítrico es una característica importante por su relación significativa con rendimiento, debido a que está relacionado con la sanidad, la forma, el tamaño del grano, entre otros.

La exploración de los efectos recíprocos es importante para tomar decisiones acertadas sobre la utilización de las líneas como progenitor macho o hembra. La separación de los efectos recíprocos en efectos maternos o extra nucleares, pero de naturaleza genética y los no maternos o de naturaleza epigenética nos indican que factores son los que están involucrados en la expresión de un carácter como efectos recíprocos (Hernández et al., 2018).

\section{EREC, EMAT y ENMAT}

De acuerdo con la información presentada en el Cuadro 2, la fuente de variación de efectos recíprocos en lo general no mostró significancia para las variables estudiadas, indicando que el aprovechamiento de las cruzas podría hacerse en ambos sentidos (directa o recíproca) sin tener cambios significativos en el comportamiento de la descendencia. Kang et al. (1999), reportan que la división del efecto recíproco (EREC) en efectos maternos (EMAT) y no maternos (ENMAT) en el análisis dialélico, es útil para determinar si factores maternales o extra nucleares están involucrados en la expresión de un carácter. Estos efectos citoplasmáticos presentes en las cruzas recíprocas influyen en algunas características; además, los efectos recíprocos pueden extenderse ampliamente y la expresión fenotípica de los caracteres puede deberse a efectos genéticos y citoplasmáticos.

En el Cuadro 4, se puede observar de manera específica, que la cruza LE03xLE01 presenta significancia a $p \leq 0.05$ para EREC, esta cruza muestra una reducción en profundidad de grano al cambiar la participación del orden de los progenitores, indicativo de que para estas variables es mejor utilizar la cruza de forma LE01xLE03 para tener descendencia con mayor profundidad de grano. 
Cuadro 4. Estimación de efectos recíprocos, maternos y no maternos, obtenidos a través del diseño dialélico de Griffing modelo II, método III de cuatro variables agronómicas en 10 cruzas en $F_{1}$.

\begin{tabular}{|c|c|c|c|c|}
\hline Efectos & $\begin{array}{l}\text { Longitud de mazorca } \\
\qquad(\mathrm{cm})\end{array}$ & $\begin{array}{c}\text { Peso hectolítrico } \\
\text { (vol) }\end{array}$ & $\begin{array}{l}\text { Profundidad de grano } \\
\qquad(\mathrm{cm})\end{array}$ & $\begin{array}{l}\text { Rendimiento } \\
\left(\mathrm{t} \mathrm{ha}^{-1}\right)\end{array}$ \\
\hline \multicolumn{5}{|l|}{$\begin{array}{l}\text { Efectos } \\
\text { recíprocos }\end{array}$} \\
\hline LE02xLE01 & 0.451 & -1.013 & -0.034 & 0.1962 \\
\hline LE03xLE01 & 0.655 & 0.588 & $-0.759^{* *}$ & $2.2925^{* *}$ \\
\hline LE04xLE01 & -0.533 & 1.063 & -0.05 & 0.02 \\
\hline LE05xLE01 & 0.945 & 0.45 & 0.078 & 0.24 \\
\hline LE03xLE02 & 0.32 & -0.25 & -0.085 & 0.4462 \\
\hline LE04xLE02 & -0.2 & -0.488 & -0.046 & -0.5462 \\
\hline LE05xLE02 & -0.21 & -1.05 & 0.028 & -0.7912 \\
\hline LE04xLE03 & 0.983 & 0.375 & 0.049 & -0.255 \\
\hline LE05xLE03 & 0.613 & -0.1 & -0.018 & -0.3825 \\
\hline LE05xLE04 & 0.613 & -1.388 & 0.085 & 0.175 \\
\hline \multicolumn{5}{|l|}{$\begin{array}{l}\text { Efecto } \\
\text { materno }\end{array}$} \\
\hline LE01 & 0.304 & 0.2175 & -0.153 & 0.5497 \\
\hline LE02 & -0.108 & -0.155 & -0.014 & -0.2175 \\
\hline LE03 & 0.124 & -0.0125 & 0.175 & -0.6752 \\
\hline LE04 & 0.091 & -0.4675 & 0.0265 & 0.1912 \\
\hline LE05 & -0.411 & 0.4175 & -0.0345 & 0.1517 \\
\hline \multicolumn{5}{|l|}{$\begin{array}{c}\text { Efecto no } \\
\text { materno }\end{array}$} \\
\hline LE02xLE01 & 0.039 & -1.385 & 0.1053 & -0.571 \\
\hline LE03xLE01 & 0.475 & 0.358 & $-0.4308^{*}$ & 1.0675 \\
\hline LE04xLE01 & -0.745 & 0.378 & 0.1295 & -0.3385 \\
\hline LE05xLE01 & 0.231 & 0.65 & 0.196 & -0.158 \\
\hline LE03xLE02 & 0.552 & -0.108 & 0.104 & -0.0115 \\
\hline LE04xLE02 & -0.001 & -0.8 & -0.0058 & -0.1375 \\
\hline LE05xLE02 & -0.512 & -0.478 & 0.007 & -0.422 \\
\hline LE04xLE03 & 0.95 & -0.08 & -0.0998 & 0.6115 \\
\hline LE05xLE03 & 0.078 & 0.33 & -0.227 & 0.4445 \\
\hline LE05xLE04 & 0.204 & -0.503 & 0.024 & 0.1355 \\
\hline
\end{tabular}

${ }^{*},{ }^{* *}=$ significativo a $(p \leq 0.05)$ y $(p \leq 0.05)$ respectivamente; LE01= línea enana $01 ;$ LE02= línea enana 02; LE03= línea enana 03; LE04= línea enana 04; LE05= línea enana 05. 
La variable de rendimiento presenta significancia al $p \leq 0.05$ en esta misma cruza; esto indica que, para la magnitud de la expresión de un carácter en una cruza, es importante determinar si es con la directa o con la recíproca con la que se obtiene la expresión más deseable del carácter (Hernández et al., 2018). Los efectos no maternos considerados como efectos epigenéticos, según Morange (2006), son producto de la interacción entre el organismo, su material genético (incluyendo genes y materiales no codificantes) y el ambiente durante el desarrollo, de modo que el modelo permite representar las influencias ambientales en el desarrollo y la expresión génica.

De acuerdo con lo que menciona Morange (2006) en el párrafo anterior, podemos deducir la importancia de este efecto para la cruza LE03xLE01 que presenta significancia al $p \leq 0.05$ en la variable de profundidad de grano, como lo mencionan King et al. (2007); Barca et al. (2014) dichos efectos se presentan por estrés generado por sequía, bajas temperaturas, salinidad, etc. No se aprecian diferencias significativas para el efecto materno en ninguna de las variables evaluadas.

Por último, del Cuadro 2, las fuentes de variación que abordan las interacciones se destacan que Hib x Amb en las variables longitud de mazorca, profundidad de grado y rendimiento, no mostraron significancia, el comportamiento de estas variables no cambia entre los ambientes; es decir, el valor promedio de las variables permanece estable a través de los ambientes. Caso contrario a lo anterior, para la variable de peso hectolítrico que presentó efectos significativos al $(p \leq 0.05)$ indicando que esta variable no es estable ya que cambia de orden a través de ambientes.

En las interacciones ACG x Amb, no se detectaron diferencias significativas, es decir la estimación de ACG en los genotipos involucrados permanecen estables a través de ambientes, al respecto mencionaremos que si la interacción de ACG x Amb fuese significativa indicaría la desviación genotípica esperada de los valores de los parentales, causada por el ambiente, que resulta de interés para el fitomejorador, ya que si esta interacción es alta (mayor que la ACG), los estimadores de heredabilidad son sesgados. Lo ideal, es que el valor de la ACG debería ser el mismo para cada genotipo en los diferentes ambientes; es decir, que la correlación entre ACG y genotipo fuera igual a uno. Al igual que la interacción anterior las de ACE x Amb, EREC x Amb, EMAT x Amb, ENMAT x Amb, también fueron no significativas, es decir los efectos permanecen estables a través de ambientes.

\section{Efectos genéticos en porcentaje}

Se consideró importante explorar la aportación de los efectos genéticos en cada una de las variables estudiadas por estar en relación directa con la heredabilidad y su comportamiento genético (Cuadro 5). Los resultados indican, que la ACG contribuyó con $66 \%$ a la suma de cuadrados en la variable de longitud de mazorca, 21\% para profundidad de grano, 23\% para peso hectolítrico y $65 \%$ para rendimiento, dándonos una idea clara de que longitud de mazorca y rendimiento tendrán una mayor heredabilidad comparada entre las variables estudiadas, tal como se comprueba en el (Cuadro 6). Estos resultados concuerdan con los encontrados por Al-Naggar y Atta (2014); Kamara et al. (2020). El efecto de ACE obtuvo mayor contribución en la expresión del peso hectolítrico aportando $47 \%$, esto implica que este carácter está controlado principalmente por efectos no aditivos o de dominancia. 
Cuadro 5. Porcentaje de contribución de la acción génica en cuatro variables agronómicas.

\begin{tabular}{lcccc}
\hline Variables & $\begin{array}{c}\text { Longitud de mazorca } \\
(\mathrm{cm})\end{array}$ & $\begin{array}{c}\text { Peso hectolítrico } \\
(\mathrm{vol})\end{array}$ & $\begin{array}{c}\text { Profundidad de grano } \\
(\mathrm{cm})\end{array}$ & $\begin{array}{c}\text { Rendimiento } \\
\left(\mathrm{t} \mathrm{ha}^{-1}\right)\end{array}$ \\
\hline ACG & 66.82 & 21.06 & 23.17 & 65.91 \\
ACE & 6.96 & 47.86 & 25.61 & 6.62 \\
EREC & 26.21 & 31.08 & 51.22 & 27.46 \\
EMAT & 10.12 & 11.67 & 23.70 & 16.54 \\
ENMAT & 16.09 & 19.4 & 27.41 & 10.92 \\
\hline
\end{tabular}

ACG= aptitud combinatoria general; $\mathrm{ACE}=$ aptitud combinatoria específica; $\mathrm{EREC}=$ efecto recíproco; $\mathrm{EMAT}=$ efecto materno; ENMAT= efecto no materno.

El efecto recíproco tiene efectos relevantes para la variable profundidad de grano lo cual es indicativo de que hay cruzas en la que los progenitores no se comportan de igual manera en la cruza directa que en la cruza recíproca, atribuible a que existen genes extra nucleares que influyen en la expresión de esta variable, tal como se detectó en la cruza LE03xLE01 del (Cuadro 4).

Lo anterior se traduce a que el comportamiento de las cruzas cambia dependiendo el orden de los progenitores macho o hembra. Al explorar los porcentajes de contribución que tienen los efectos recíprocos en el fenotipo de los materiales evaluados, se observó que el efecto no materno (ENMAT) es de mayor importancia en tres de las variables. Sámano et al. (2009) reportaron que al combinar líneas entre dos grupos germoplásmicos no emparentados genéticamente, el orden de participación de los progenitores cambia la expresión genética y fenotípica de la $\mathrm{F}_{1}$; es decir, existen efectos recíprocos.

\section{Varianza genética y heredabilidad}

Para entender mejor la presencia de valor reproductivo en las variables, se calculó la heredabilidad en sentido estrecho (Cuadro 6), con esto se logra medir el grado de diferencia entre las poblaciones debido al efecto de los genes. La varianza aditiva resulto importante para longitud de mazorca y rendimiento lo que le permitió, tener heredabilidad de 0.569 y 0.581 ; por estos valores se recomienda utilizar el método de mejoramiento de selección recurrente de hermanos completos. Al-Naggar y Atta (2017), mencionan que la aditividad es importantes en la heredabilidad del rendimiento de grano y otros rasgos agronómicos.

Cuadro 6. Componentes de varianza genética y heredabilidad en cuatro variables agronómicas.

\begin{tabular}{ccccc}
\hline $\begin{array}{c}\text { Longitud de mazorca } \\
(\mathrm{cm})\end{array}$ & $\begin{array}{c}\text { Peso hectolítrico } \\
(\text { vol })\end{array}$ & $\begin{array}{c}\text { Profundidad de grano } \\
(\mathrm{cm})\end{array}$ & $\begin{array}{c}\text { Rendimiento } \\
\left(\mathrm{t} \mathrm{ha}^{-1}\right)\end{array}$ \\
\hline$\sigma_{A}^{2}$ & 1.907 & -0.271 & 0.009 & 8.93 \\
$\sigma_{D}^{2}$ & 0.023 & 1.253 & -0.015 & -0.157 \\
$\sigma_{F}^{2}$ & 3.349 & 2.239 & 0.125 & 15.371 \\
$\mathrm{~h}^{2}$ & 0.569 & -0.121 & 0.073 & 0.581 \\
\hline
\end{tabular}

$\sigma_{A}^{2}=$ varianza aditiva; $\sigma_{D}^{2}=$ varianza de dominancia; $\sigma_{F}^{2}=$ varianza fenotípica; $\mathrm{h}^{2}=$ heredabilidad. 
Los estimados de heredabilidad para peso hectolítrico y profundidad de grano fueron de $-0.121 \mathrm{y}$ 0.073 esto indica que el incremento de los alelos favorables para estos componentes dentro de las poblaciones se hace a tasas lentas y por ende, el progreso genético es mucho menor. En la variable de peso hectolítrico y de profundidad de grano es factible utilizar métodos de mejoramiento de progenies endogámicas por lo menos en dos ciclos de selección y así, aumentar la varianza aditiva y depurar la carga genética y posteriormente, seguir con selección recurrente familiar. Silva et al. (2018), indican que la función más importante de la heredabilidad en estudios genéticos de caracteres cuantitativos es su rol predictivo para indicar la confiabilidad del valor fenotípico como guía del valor reproductivo. Caracteres con heredabilidad alta pueden fácilmente ser fijados por selección familiar resultando en elevada ganancia genética.

\section{Conclusiones}

El efecto de tipo aditivo es importante para las variables de longitud de mazorca y rendimiento y efectos del tipo no aditivo son los responsables del comportamiento fenotípico de peso hectolítrico. Sin embargo, los efectos recíprocos juegan un papel muy importante en la expresión fenotípica de profundidad de grano, atribuible tanto a efectos maternos como no maternos.

La heredabilidad estimada para longitud de mazorca y rendimiento tiene un valor alto (0.569 y 0.581) respectivamente, mientras que para peso hectolítrico y profundidad de grano la heredabilidad es baja (-0.121 igual a 0 y 0.073$)$ respectivamente, lo que sugiere que para las primeras dos variables será muy eficiente un esquema de mejoramiento de hermanos completos y para las otras dos variables se requiere mejoramiento bajo progenies endogámicas para tratar de elevar la varianza aditiva y mejorar la heredabilidad.

\section{Literatura citada}

Abbott, L. y Pistorale, S. 2011. Análisis de la estabilidad y adaptabilidad de caracteres de interés agronómico en genotipos selectos de cebadilla criolla (Bromus catharticus). Agriscientia. 28(2):109-117. http://www.scielo.org.ar/pdf/agrisc/v28n2/v28n2a02.pdf.

Al-Naggar, A. M. M; Shabana, R. and Atta, M. M. M. 2014. Heterosis and type of gene action for some adaptive traits to high plant density in maize. Egyptian J. Plant Breed. 18(2):189-209.

Al-Naggar, A. M. M. and Atta, M. M. M. 2017. Elevated plant density effects on performance and genetic parameters controlling maize (Zea mays L.) agronomic traits. J. Adv. Biol. Biotechnol. 12(1):1-20. https://doi.org/10.9734/JABB/2017/31550.

Arellano, V. J. L.; Gámez, V. A. J. y Ávila, P. M. A. 2010. Potencial agronómico de variedades criollas de maíz cacahuacintle en el Valle de Toluca. Rev. Fitotec. Mex. 33(4):37-41. http://www.scielo.org.mx/pdf/rfm/v33nspe4/v33ns pe4a9.pdf.

Barca, H. J.; Collado, M. B.; Aulicino, M. B.; Arturi, M. y Molina, M. C. 2014. Cambios epigenéticos en maíz bajo estrés salino. Argentina. J. Basic Appl. Gen. 25(1):107.

Bernardo, R. 2001. Breeding potential of intra and interheterotic group crosses in maize. Crop Sci. 41(1):68-71.

Borrás, L. and Otegui, M. E. 2001. Maize kernel weight response to postflowering source-sink ratio. Crop Physiol. Metabolism. 41(6):1816-1822. 
Coutiño, E. B.; Vidal, M. V. A.; Cruz, G. B. y Cruz, V. C. 2010. Aptitud combinatoria general y específica del contenido de azúcares en maíces criollos eloteros. Rev. Fitotec. Mex. 33(4):57-61. http://www.scielo.org.mx/pdf/rfm/v33nspe4/v33nspe4a12.pdf.

De León, C. H.; Rincón, S. F.; Reyes, V. M. H.; Sámano, G. D.; Martínez, Z. G.; Cavazos, C. R. y Figueroa, C. J. D. 2005. Potencial de rendimiento y estabilidad de combinaciones germoplásmicas formadas entre grupos de maíz. México. Rev. Fitotec. Mex. 28(2):135143. http://www.revistafitotecniamexicana.org/documentos/28-2/7a.pdf.

Díaz, C. T. G.; Sabano, A. F. A.; Zambrano, M. S. y Vasconez, M. H. G. 2009. Evaluación productiva y calidad del grano de cinco híbridos de maíz (Zea mays L.) en dos localidades de la provincia de Los Ríos. Ciencia y Tecnología. 2(1):15-23. https://dialnet.unirioja.es/ servlet/articulo?codigo $=4053227$.

Guillen, C. P.; De La Cruz, L. E.; Castañón, N. G.; Osorio, O. R.; Brito, M. N. P.; Lozano, del R. A. J. y López, N. U. 2009. Aptitud combinatoria general y específica de germoplasma tropical de maíz. Trop. Subtrop. Agroecosy. 10(1):101-107. https://www.redalyc.org/ pdf/939/93911243010.pdf.

Griffing, B. 1956. Concept of general and specific combining ability in relation to diallel crossing systems. Division of Plant Industry, CSIRO. Canberra, ACT. 463-493 pp. https://www.publish.csiro.au/bi/pdf/bi9560463.

Haochuan, L.; Jihua, H. T.; Yanmin, Y. J. and Zonghua, L. 2014. Analysis on combining ability and estimation of genetic parameters for chlorophyll content in maize. Plant Breed. Crop Sci. 6(8):97-104.

Hernández, A. E.; De León, C. H.; Zamora, V. V. M.; Hernández, S. J. L. y Cahuare, R. C. 2018. Exploración genética y reconstitución de una población de maíz enano. Agraria. 15(2):67-78.

Hermes, A. T.; Espitia, C. M. y Cardona, A. C. 2017. Adaptabilidad y estabilidad fenotípica en cultivares de fríjol caupí en el caribe húmedo colombiano. Biotecnología en el Sector Agropecuario y Agroindustrial. 15(2):14-22. http://www.scielo.org.co/pdf/bsaa/ v15nspe2/1692-3561-bsaa-15-spe2-00014.pdf.

Kamara, M. M.; Rehan, M.; Ibrahim, K. M.; Alsohim, A. S.; Elsharkawy, M. M.; Kheir, A. M. S.; Hafez, E. M. and El-Esawi, M. A. 2020. Genetic diversity and combining ability of white maize inbred lines under different plant densities. Plants. 9(9):1-23. https://doi.org/10.3390/plants9091140.

Kang, M. S.; Kushairi, D. A.; Zhang, Y. and Magari, R. 1999. Combining ability for rind puncture resistance in maize. Crop Breed. Gen. Cytol. 39(2):368-371. https://doi.org/10.2135/ cropsci1999.0011183X0039000200011x.

King, R. C.; Stansfield, W. D. and Mulligan, P. K. 2007. A dictionary of genetics. Oxford University Press. $7^{\text {th }}$ (Ed). New York. 608 p. Doi: 10.1093/acref/9780195307610.001.0001.

Lobato, O. R.; Molina, G. J. D.; López, R. J. J.; Mejía, C. J. A. y Reyes, L. D. 2010. Criterios para elegir el mejor probador de la aptitud combinatoria general para rendimiento de grano de líneas autofecundadas de maíz. Agrociencia. 44(1):17-30. http://www.scielo.org.mx/pdf/ agro/v44n1/v44n1a2.pdf.

Luna, O. J.; García, H. J. L.; Valdés, C. R. D.; Gallegos, R. M. A.; Preciado R. P.; Guerrero, G. C. y Espinoza, B. A. 2013. Aptitud combinatoria y sus componentes genéticos en líneas de maíz. Universidad y Ciencia. 29(3):243-253. http://www.scielo.org.mx/pdf/uc/v29n3/ v29n3a3.pdf. 
Morange, M. 2006. The relations between genetics and epigenetics. A historical point of view. Annals of the New York Academy of Sciences. 981(1):50-60. Doi: 10.1111/j.1749-6632. 2002.tb04911.x.

Olán, O. M.; Espitia, R. E.; López, S. H.; Villaseñor, M. H. E.; Peña, B. R. J. y Herrera, H. J. 2012. Calidad física de grano de trigos harineros (Triticum aestivum L.) mexicanos de temporal. Rev. Mex. Cienc. Agríc. 3(2):271-283. http://www.scielo.org.mx/pdf/remexca/v3n2/ v3n2a5.pdf.

Pecina, M. J. A.; Mendoza, C. M. C.; López, S. J. A.; Castillo, G. F.; Mendoza, R. M. y Ortiz, C. J. 2011. Rendimiento de grano y sus componentes en maíces nativos de Tamaulipas evaluados en ambientes contrastantes. Rev. Fitotec. Mex. 34(2):85-92. http://www.scielo.org.mx/pdf/rfm/v34n2/v34n2a6.pdf.

Reyes, L. D.; Molina, G. J. D.; Oropeza, R. M. A. y Moreno, P. E. C. 2004. Cruzas dialélicas entre líneas autofecundadas de maíz derivadas de la raza tuxpeño. Rev. Fitotec. Mex. 27(1):49-56. https://www.revistafitotecniamexicana.org/documentos/27-1/7a.pdf.

Ruales, E. F. R.; Manrique, P. C. y Cerón, M. M. 2007. Fundamentos en mejoramiento animal. Vieco. $1^{\text {ra }}$ (Ed.). Medellín. 208 p.

Sámano, G. D.; Rincón, S. F.; Ruíz, T. N. A.; Espinoza, V. J. y De León, C. H. 2009. Efectos genéticos en cruzas directas y recíprocas formadas a partir de líneas de dos grupos germoplásmicos de maíz. Rev. Fitotec. Mex. 32(1):67-74. http://www.scielo.org.mx/pdf/ $\mathrm{rfm} / \mathrm{v} 32 \mathrm{n} 1 / \mathrm{v} 32 \mathrm{n} 1 \mathrm{a} 9 . \mathrm{pdf}$.

SIAP. 2020. Servicio de Información Agrícola y Pecuaria. Anuario estadístico de la producción agrícola. https://nube.siap.gob.mx/cierreagricola/.

Silva, D. R.; García, M. P.; Faleiro, S. D. y López S. C. 2018. Determinación de componentes de la varianza y parámetros genéticos en una población segregante de maíz tropical. Bioagro. 30(1):67-77. http://ve.scielo.org/scielo.php?script=sci_arttext\&pid=S1333612018000100 $007 \& \operatorname{lng}=$ es\&tlng=es.

Wong, R. R.; Gutiérrez, D. R. E.; Palomo, G. A.; Rodríguez, S. H.; Córdoba, H. O.; Espinoza, B. A. y Lozano, J. J. G. 2007. Aptitud combinatoria de componentes del rendimiento en líneas de maíz para grano en la comarca lagunera. México. Rev. Fitotec. Mex. 30(2):181-189. https://www.revistafitotecniamexicana.org/documentos/30-2/10a.pdf.

Zhang, Y.; Kang, S. M. and Lamkey, K. R. 2005. DIALLEL-SAS05: a comprehensive program for Griffing's and Gardner-Eberhart analyses. Agron. J. 97(4):1097-1106. Doi: 10.2134/agroni2004.0260. 